\title{
Network Traffic Prediction Based on LMD and Neural Network LUO Yongsheng
}

\author{
Liaoning Geology Engineering Vocational College, Liaoning Dandong, 118000, China \\ lys10@163.com
}

Keywords: network traffic; LMD; wavelet neural network; prediction

\begin{abstract}
The network traffic data has a very strong burst and self similarity and so on, which results in the system flow is not stable and inaccurate. The traditional network traffic prediction model is not accurate enough. To solve the above problems, a network traffic prediction model based on local mean decomposition (LMD) and wavelet neural network (WNN) was proposed. The original network traffic data is decomposed into a series product function (PF), which can reflect the fractal characteristic of the network traffic data. The PFs are taken as the input of the WNN to establish the prediction model. In this method, the network traffic prediction model can make full use of the inherent characteristics of the network traffic data, and get more accurate prediction results. Experimental data processing results show that the prediction accuracy of the network traffic prediction model based on LMD and WNN is higher than the traditional models.
\end{abstract}

\section{Introduction}

With the rapid development of the scale of the communication network and the business increasing, the network management is becoming more and more important. The network management is gradually developing from running management to performance and business management. Furthermore, the management mode is developed from the automation management to the intelligent management. Therefore, intelligent analysis of the performance of the network is one of the problems to be solved at present. Meanwhile, because the network is more and more complex, the possibility of the occurrence of all kinds of failure is also greater and the network performance is more likely to be affected. All of the above reasons lead to the difficulty of network management. In order to provide users with quality services, network maintenance and management is particularly important. The network traffic prediction technology can establish an appropriate prediction model based on the historical data to predict the future traffic data [1]. According to the results of the prediction, the trend of the network traffic in the future can be obtained. If the result of the prediction is beyond the preset threshold, the alarm will be issued so that the network administrator can check the network status and avoid the occurrence of network failures. Therefore, network traffic prediction can provide effective basis for network bandwidth allocation, traffic control, routing control, admission control and the security management. The network traffic prediction mainly has two kinds of prediction algorithms: traditional statistical methods and intelligent prediction methods [2]. The traditional statistical methods include regression prediction method, multivariate statistical method and time series model method. The intelligent prediction methods include support vector machine (SVM) and neural network method, etc. The traditional statistical methods are based on linear modeling, which is difficult to accurately describe the nonlinear relationship between the network traffic and the impact of history. Therefore, the prediction results of the traditional statistical methods are not reliable [3]. The intelligent prediction methods can get higher prediction accuracy. However, the intelligent prediction methods has some shortages, such as slow convergence rate, poor generalization ability and easy to fall into local minimum value, etc. As the network scale increases gradually, the network traffic has the characteristic of randomness, periodicity and abrupt change, which results in the traditional network traffic prediction models have a certain gap with the actual need.

To overcome the shortages of the traditional network traffic prediction model, an improved network traffic prediction mode based on LMD and WNN was proposed. The historical data of 
network traffic is decomposed by LMD to obtain a series of PFs. The PFs can reflect the characteristic of the network traffic at different levels. The prediction model is established by WNN and the PFs are acted as the input of WNN. This method can make full use of the historical data of the network traffic, and it is more suitable for the randomness, periodicity and abrupt change of the network traffic. Therefore, the network traffic prediction based on LMD and WNN can obtain more accurate prediction results.

\section{The basic principle of LMD}

LMD is an adaptive signal decomposition method [4] which decomposes a signal to a series of PFs, every PF corresponding to a product of an envelope signal and a pure FM signal. The implement step of LMD can be summarized as follows:

Step1: Determine all the local extreme points of the signal $x(t)$, including the maxima and minima $n_{11}\left(k_{l}\right)(l=1,2, \cdots, m)$, local amplitude function $m_{11}(t)$ and local mean function $a_{11}(t)$ can be computed by

$$
\begin{aligned}
m_{11}(t) & =\frac{n_{11}\left(k_{l}\right)+n_{11}\left(k_{l+1}\right)}{2} \\
a_{11}(t) & =\frac{\left|n_{11}\left(k_{l}\right)-n_{11}\left(k_{l+1}\right)\right|}{2}
\end{aligned}
$$

where $k_{l}=k_{1}, k_{2}, \cdots, k_{M-1}, t \in\left[k_{l}, k_{l+1}\right]$.

Step2: Adopt the sliding average for all the local mean function and local amplitude function for smoothing. Then subtract the smoothing local mean function $\tilde{m}_{11}(t)$ from the original signal $x(t)$, and we get the separation signal $h_{11}(t)$. Normalize $h_{11}(t)$ by smooth local amplitude function $\tilde{a}_{11}(t)$ is given by

$$
\begin{aligned}
& h_{11}(t)=x(t)-\tilde{m}_{11}(t) \\
& s_{11}(t)=h_{11}(t) / \tilde{a}_{11}(t)
\end{aligned}
$$

Step3: if $s_{11}(t)$ is not a pure FM signal in [-1,1], take $s_{11}(t)$ as the original signal and repeat step1 to step2 till the separation signal meet the condition. Instantaneous phase and instantaneous frequency can be determined by

$$
\begin{aligned}
\varphi_{1}(t) & =\arccos \left(s_{11}(t)\right) \\
f_{1}(t) & =d \varphi_{1}(t) / 2 \pi d t
\end{aligned}
$$

Step4: Use all the local amplitude function product to obtain instantaneous amplitude is given by

$$
a_{1}(t)=a_{11}(t) a_{12}(t) \cdots a_{1 n}(t)=\prod_{q=1}^{n} a_{1 q}(t)
$$

The first PF is given by

$$
P F_{1}(t)=a_{1}(t) s_{1 n}(t)
$$

Step5: Here separate the first PF from the original signal as follow

$$
u_{1}(t)=x(t)-P F_{1}(t)
$$

Then let $u_{1}(t)$ is a new original signal and repeat step1 to step4 till all $u_{k}(t)$ meet the monotone function condition. Thus, the original signal can be expressed as the sum of PF components and a monotonic component

$$
x(t)=\sum_{p=1}^{k} P F_{p}(t)+u_{k}(t)
$$




\section{The prediction model of WNN}

WNN is the combination of wavelet transform and NN [5] which can be divided into loose type and compact type. Compared with $\mathrm{BP} \mathrm{NN}$, WNN has faster convergence rate and higher convergence precision. The three layer wavelet neural network is taken as an example to illustrate the prediction model as show in Fig. 1. Where $w_{i j}(i=1,2, \cdots, m ; j=1,2, \cdots k)$ is the weight between the input layer and the hidden layer and $w_{j}$ is the weight between the hidden layer and the output layer. According to the network traffic prediction model, the output layer of the WNN only has one neuron.

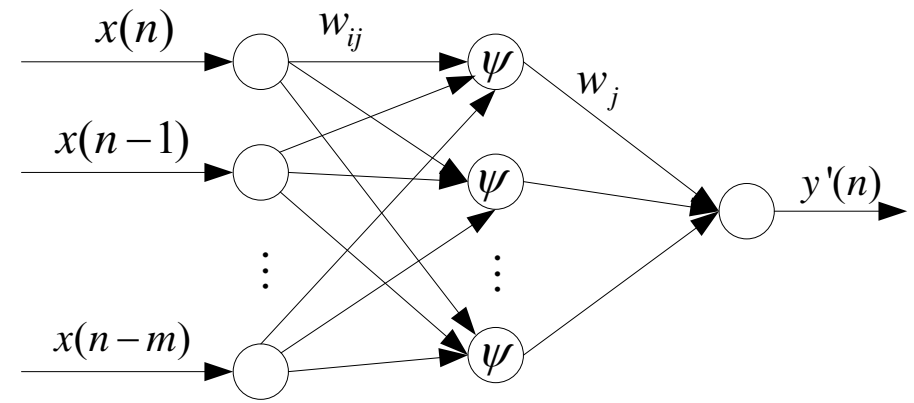

Fig.1 Three layer wavelet neural network structure diagram

Let the input of the hidden layer is $u_{j}(n)$ and the output is $I_{j}(n)$. The input of the output layer is $v(n)$ and the output is $y^{\prime}(n)$. According to the transmission formula of the network, the state equation of the WNN is obtained as follows:

$$
\begin{aligned}
u_{j}(n) & =\sum_{i=1}^{m} w_{i j}(n) x(n-i) \\
I_{j}(n) & =\psi_{a, b}\left[u_{j}(n)\right] \\
v(n) & =\sum_{j=1}^{k} w_{j}(n) I_{j}(n) \\
y^{\prime}(n) & =f[v(n)]
\end{aligned}
$$

where $f($.$) is the transfer function of the output layer. \psi_{a, b}(\cdot)$ is the wavelet function which carries the wavelet transform of the output signal of the hidden layer. In this paper, we choose the Morlet wavelet generating function which is given by

$$
\begin{gathered}
\psi(x)=x e^{-\frac{1}{2} x^{2}} \\
\psi_{a, b}(x)=|a|^{\frac{1}{2}} \psi\left(\frac{x-b}{a}\right)=|a|^{\frac{1}{2}} \frac{x-b}{a} e^{-\frac{(x-b)^{2}}{2 a^{2}}}
\end{gathered}
$$

where $b$ is the shift factor and $a$ is the scale factor of wavelet transform.

The proposed network traffic prediction model based on LMD and WNN takes the advantages of LMD and WNN respectively and the model can be shown in Fig.2.

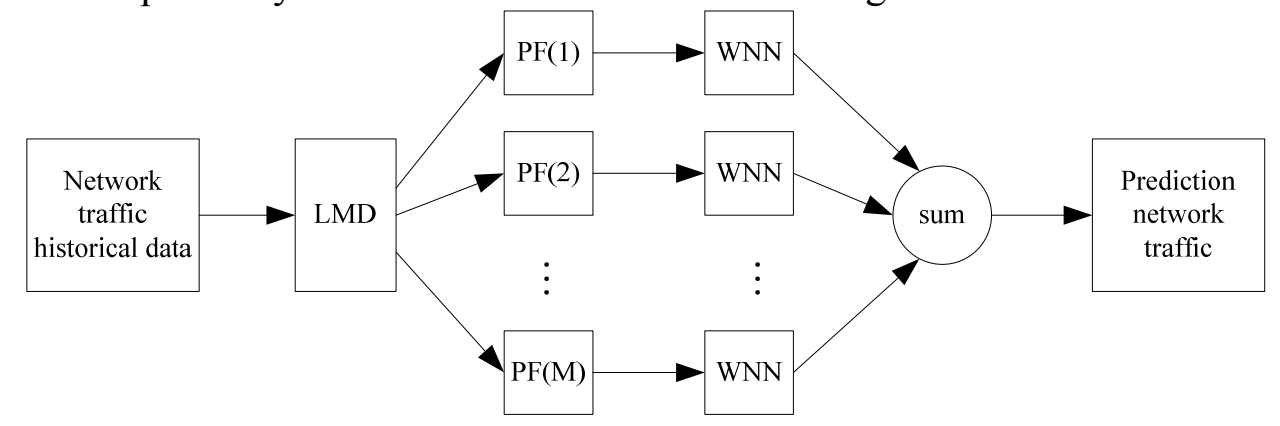

Fig.2 The model of network traffic prediction 
The network traffic historical data is decomposed by LMD to obtain a series PFs. Where $P F(i)$ is the product function $(i=1,2, \cdots M) . M$ is the maximum decomposition level of LMD. Different PF reflects the different characteristics of network traffic. Therefore, the characteristic of the PF is simpler than the network traffic historical data. By using the PF as the input of WNN for prediction model establishing, we can obtain more accurate prediction. Meanwhile, this method can deal with some mutation data in the network traffic historical data.

\section{Experimental data processing and analysis}

Network traffic experiment data is collected from a server. A total of 200 data were collected, which is shown in Fig.3. Using the first 100 data to form a training sample for WNN training and the rest of the 100 data is used to test the model. LMD decomposition level is 5 . The prediction error is shown in Fig.4. From the results, we can see that the method proposed in this paper has higher prediction accuracy.

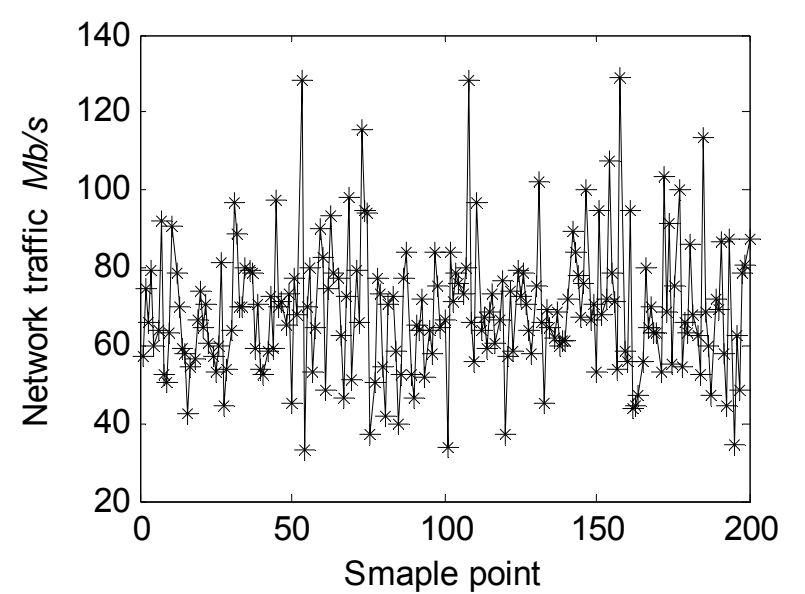

Fig. 3 the network traffic historical data

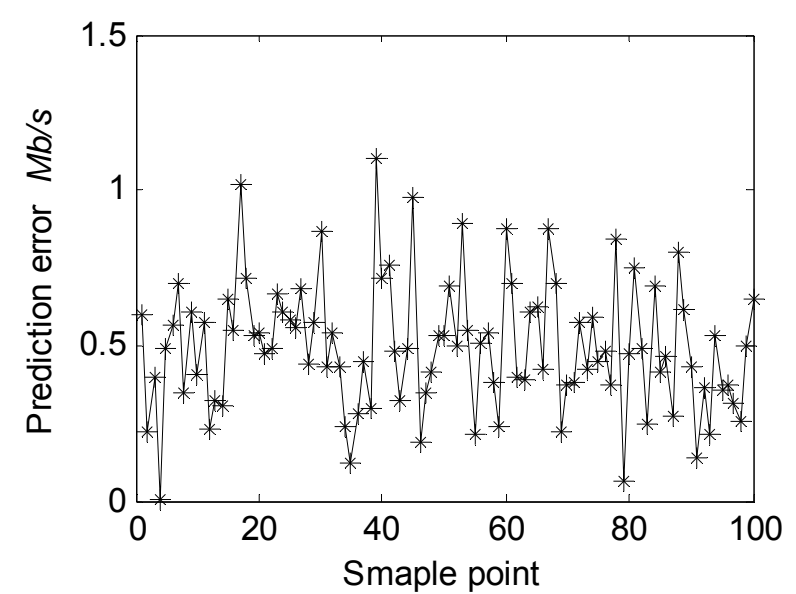

Fig.4 The prediction error

\section{Conclusion}

This work proposed a network traffic prediction method based on LMD and WNN. By using LMD to decompose the historical data of network traffic, the characteristic of the data can be extracted. Based on this, WNN is trained according to the PFs to obtain high prediction accuracy. The experimental data processing result show the effectiveness of the proposed method.

\section{References}

[1] W. ZENG. Network traffic prediction based on SVM optimized by multi-subpopulaiton particle swarm optimization algorithm [J]. Journal of Beijing Jiaotong University, 2013, 37(5): 62-66.

[2] T.T. THUY, Grenville Armitage. A survey of techniques for internet traffic classification using machine learning [J]. IEEE Communication Surveys and Tutorials, 2008, 10(4): 56-76.

[3] Z. ZHANG. Network traffic prediction based on combination neural network [J]. Microelectronics \& Computer, 2012, 29(3): 98-101+105.

[4] J.S. CHENG, M.L. SHI, Y. YANG. Roller bearing fault diagnosis method based on LMD and neural network [J]. Journal of Vibration and Shock, 2010, 29(8): 41-44.

[5] W. HUANG, Y. HE, H. XIA. Internet traffic prediction based on wavelet neural network [J]. Computer Science, 2011, 38(10A): 296-298+330. 